\title{
Dermoscopy of accessory nipples in authors' own study
}

\author{
Grażyna Kamińska-Winciorek', Jan Szymszal², Wojciech Silny ${ }^{3}$ \\ ${ }^{1}$ Center for Diagnostics and Treatment of Skin Diseases, Katowice, Poland \\ Head of Center: Grażyna Kamińska-Winciorek MD, PhD \\ 2Department of Mathematics and Informatics, Higher School of Labour Safety Management, Katowice, Poland \\ Head of Department: Prof. Bohdan Mochnacki PhD \\ ${ }^{3}$ Great Poland Center of Allergology and Aesthetic Dermatology “Art Clinic”, Poznan, Poland \\ Head of Center: Prof. Wojciech Silny MD, PhD
}

Postep Derm Alergol 2014; XXXI, 3: 127-133

DOI: $10.5114 /$ pdia.2014.43189

\begin{abstract}
Introduction: The accessory nipple (AN) is characterised by its network-like structures, which may suggest the diagnosis of a melanocytic lesion. The knowledge about additional dermoscopic features of AN may greatly minimise the risk of unnecessary surgical excisions.

Aim: To analyse and present different clinical and dermoscopic forms, in which the AN may appear.

Material and methods: Ninety AN with dermoscopic features were evaluated in the study, detected in 14 patients between the years 2008 and 2014.

Results: The most common dermoscopic features of the AN were central, scar-like areas (15/19) and peripheral network-like structures (12/19). A number of cleft-like appearances (8/19) and central network-like structures (7/19) had also been observed. Moreover, among the dermoscopic features, white cobblestone-like structures (7/19), a central round dimpling with a plug (6/19) and fisheye-like structures resembling comedo-like openings (9/19) have all also been noted. There is a statistical significance in the occurrence of white cobblestone-like structures with central network-like structures (Fisher's exact test $p=0.0449$ ). The presence of peripheral network-like structures with the occurrence of central scar-like areas was statistically highly significant $(p=0.0091)$. The central round dimpling was never observed alongside any central network-like structures in any of the lesions $(p=0.0436)$.

Conclusions: Accessory nipples are most commonly characterised by the occurrence of a peripheral network-like structure accompanied by the presence of a scar-like area.
\end{abstract}

Key words: dermoscopy, dermatoscopy, accessory nipple.

\section{Introduction}

The mammary gland of an adult individual is situated on the level of the second to sixth or seventh costae, in a transverse direction towards the parasternal line, ending in the vicinity of the central axillary line [1]. Apart from the two typical mammary glands found among individuals of both genders, albeit more often among women, what is known as the mammae accesoriae, or an accessory gland, may at times be observed. There are also occurrences of an additional nipple appearing, devoid of any glands (hyperthelia). This is more commonly observed among men [1]. The frequency of the occurrence of the accessory nipple (AN) is at $1 \%$ to $2 \%$ [2]. This additional or supernumerary nipple is typically situated approximately $10 \mathrm{~cm}$ below the main nipple, and is somewhat more centrally placed on the abdomen. This nipple is usually much smaller in size and typically devoid of an areola [1] (Figure 1). The classic dermoscopic image of the normal nipple has been shown in Figure 2. Dermoscopic pictures of AN in 1 patient are presented in Figures 3 and 4.

Usually, supernumerary nipples develop along the two vertical 'milk lines' which start in the armpit on each side, run down through the standard nipple, and end at the groin [1-3]. Worldwide, only 2 cases of patients with a total of seven additional nipples have been documented [3]. The classification of the supernumerary nipple includes 8 different clinical types, according to Kajava [4]. This matter has been extensively described in the latest report published by Goyal et al. [3].

In some cases, AN (the supernumerary nipple) clinically suggest either dermatofibroma, fibroma or the presence of the melanocytic nevi [5]. The ability of recognising the AN as a potential underlying internal malignancy such as seminoma [6], renal adenocarcinoma [7] and also, poten-

Address for correspondence: Grażyna Kamińska-Winciorek MD, PhD, Center for Diagnostics and Treatment of Skin Diseases, 194/1 Armii Krajowej Av., 40-750 Katowice, Poland, phone/fax: + 48698626 208, e-mail: dermatolog.pl@gmail.com Received: 13.03.2014, accepted: 24.03.2013. 


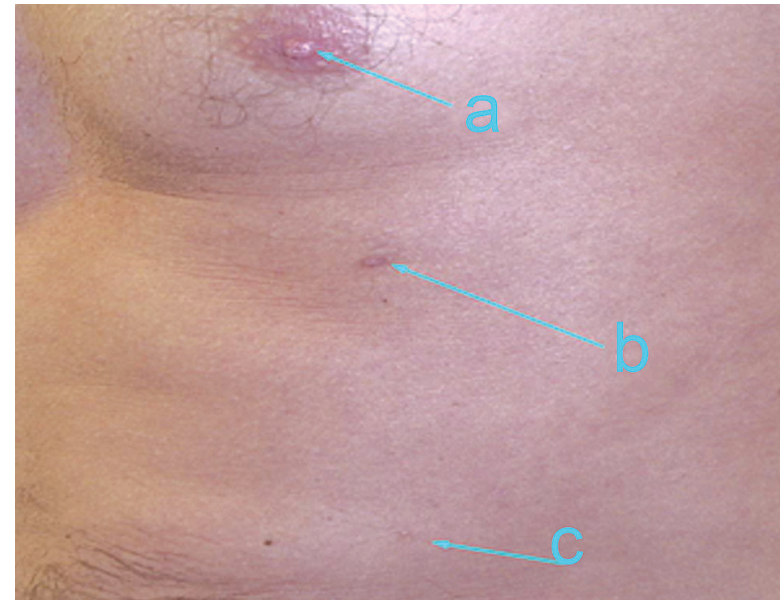

Figure 1. Clinical and dermoscopic presentation of multiple, unilateral accessory nipples. A 42-year-old patient with two accessory nipples, linearly distributed within the left milk line (b) - in the first line, (c) - in the second line, with a tendency to decline the further away the AN was situated from the normal nipple (a)

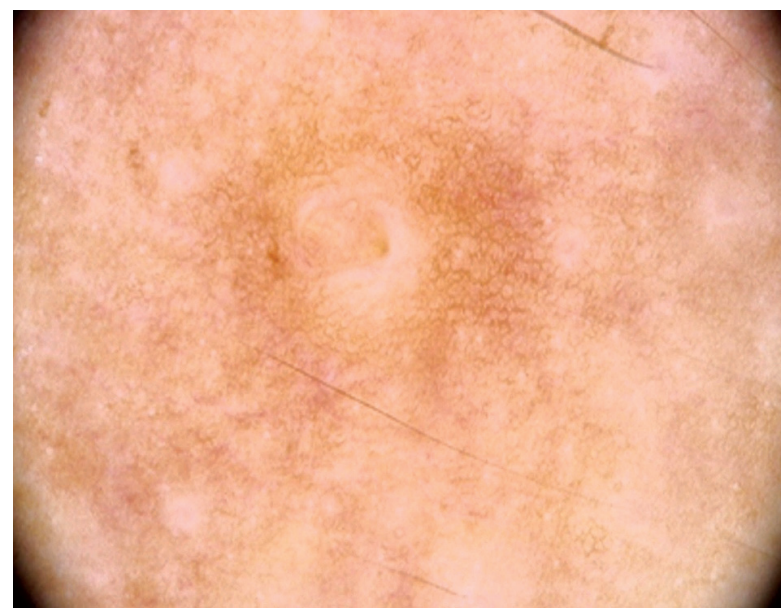

Figure 3. Dermoscopic picture of the first-line accessory nipple shows the central scar-like structureless area with a small central dimpling. Light-brown irregular peripheral network-like structures surround the central part of the accessory nipple

tially, breast cancer [8], melanoma [9] or Paget's disease [10] developing within this lesion, is absolutely crucial. Recognition of this AN as a genodermatosis with malignant potential is extremely important in all medical specialisations, such as oncology, surgery, primary care, gynaecology, dermatology and geriatrics. Therefore, the knowledge of clinical and dermoscopic recognition of the AN is a clue in the diagnostic algorithm and in the differential diagnosis of melanocytic and non-melanocytic lesions.

\section{Aim}

The aim of the study is to describe the most commonly occurring dermoscopic features of the AN, with

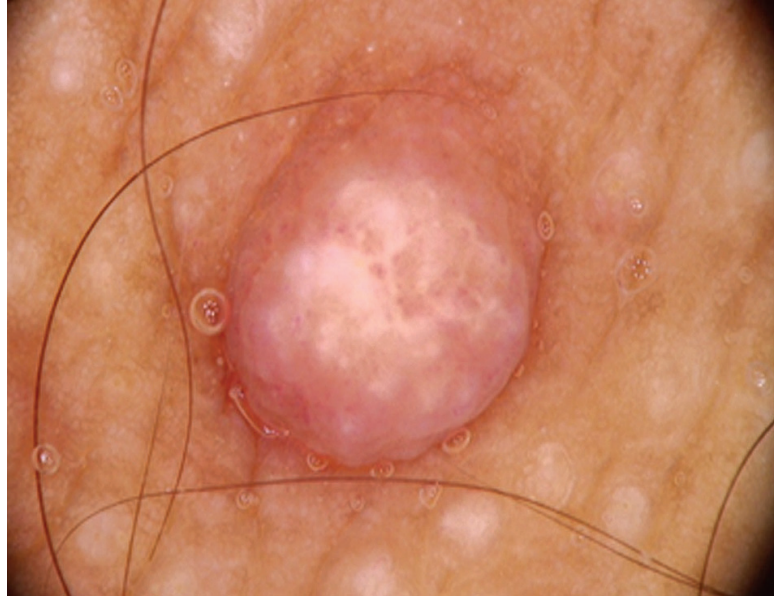

Figure 2. Dermoscopic image of a normal male nipple the central homogenous whitish and pinkish structureless area with multiple, small whitish globules corresponding to the tip of the nipple are all duly noted. Light-brownish network-like structures with several, tiny, regular, roundish globules were also observed in the areola

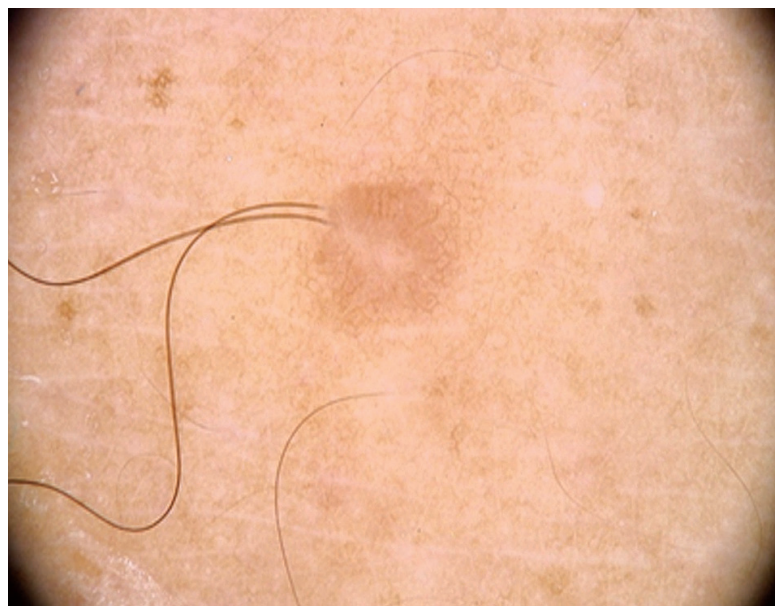

Figure 4. Dermoscopic image of the second-line accessory nipple presents a flat central scar-like area with peripheral network-like structures

their clinical manifestations. The recognition of the clinical and dermoscopic features will be helpful for doctors in consecutive diagnostic procedures.

\section{Material and methods}

Fourteen patients ( 7 males, 7 females) of an average age of 32 (the median age was 29.2, with a minimum of 14 years and a maximum of 54, SD 13.1 years) were diagnosed clinically and dermoscopically with a condition known as the AN during routine dermatologic examinations in the Private Dermatological Unit between January 2008 and January 2014. The total number of analysed dermoscopic pictures of all AN lesions was 19. The rec- 
Table 1. The definition of dermoscopic features described and observed in the conducted study, based on literature [5, 11-13], with authors' own modification

\begin{tabular}{ll}
\hline Dermoscopic feature & \multicolumn{1}{c}{ Definition } \\
\hline $\begin{array}{l}\text { Peripheral network (network-like } \\
\text { structures) }\end{array}$ & $\begin{array}{l}\text { A network of brownish, interconnected thin lines over a background of tan diffuse } \\
\text { pigmentation, located peripherally at the lesion }\end{array}$ \\
\hline Central network-like structures & $\begin{array}{l}\text { A network of brownish, interconnected thin lines over a background of tan diffuse } \\
\text { pigmentation, located centrally at the lesion }\end{array}$ \\
\hline Central white scar-like area & $\begin{array}{l}\text { Central, sharply circumscribed, round to oval, white area within the centre of an otherwise } \\
\text { regular, light-to dark brown pigmented lesion }\end{array}$ \\
\hline Cleft-like appearance & A small rim, dividing the tip into two symmetric parts \\
\hline White cobblestone-like structures & $\begin{array}{l}\text { Closely aggregated, larger, angulated whitish globules resembling cobblestones } \\
\text { Much like the classic cobblestone pattern actually consisting of pigmented globules }\end{array}$ \\
\hline Central round dimpling with a plug & Central roundish dimple filled with a darker plug \\
\hline $\begin{array}{l}\text { Fisheye-like structures (mimicking } \\
\text { comedo-like openings) }\end{array}$ & $\begin{array}{l}\text { Small, darker (brownish or black) dots visible in the form of sharply circumscribed, usually } \\
\text { round or oval structures, mimicking comedo-like openings }\end{array}$ \\
\hline
\end{tabular}

ognition of the AN was based on clinical imaging and on a palpable dimpling or tip manifested in changes located characteristically in the region where an AN might be found, as presented in the introduction.

Every AN was checked with a hand-held Delta 20 Heine dermoscope and recorded in the form of captured clinical and dermoscopic digital images with the help of the FotoFinder Dermoscope Dynamic Medicam 800 HD software (FotoFinder Systems GmbH). The recorded images were analysed retrospectively according to the procedure presented below.

All AN were described clinically and dermoscopically, taking into account their location and their overall dermoscopic pattern.

Images of all the AN were analysed according to basic dermoscopic features proposed by previous authors $[5,11-13]$ and according to our own additional criteria (Table 1):

1. Peripheral network (network-like structures),

2. Central network-like structures,

3. Central white scar-like area,

4. Cleft-like appearance,

5. White cobblestone-like structures,

6. Central round dimpling with a plug,

7. Fisheye-like structures resembling comedo-like openings.

Moreover, new dermoscopic findings observed in the course of our own research are presented in the results section.

\section{Statistical analysis}

A statistical assessment was made and implemented with the help of the Statistica StatSoft version V 7.1 PL statistic software. In this statistical analysis, the statistical level was $p<0.05$. The non-parametric Fisher's exact test was used in the statistical assessment in nominal scale. In order to compare quantitative characteristics, the parametric $t$ Student test was also adopted.

\section{Results}

Characteristics of the investigated patients' group

In the $t$ Student test, the investigated patients' group of females and males was homogeneous as regards the age criterion $(p=0.236)$. The average age for females was 36 (min. 19, max. 54 years, SD 13.3). The average age for men was 27.7 years (min. 14, max. 48, the median being 23 years, SD 12.3).

\section{Clinical presentation of accessory nipples}

The total number of bilateral nipples was 8 lesions in 4 patients. Symmetrical manifestation of the AN was observed in 2 patients with bilateral nipples (total number of AN was 4).

The unilateral clinical presentation consisted of a group of patients who were diagnosed with 9 left-sided lesions in 8 patients and 2 right-sided lesions in 2 patients.

Clinically, the AN occurred as a solitary papule in 6 lesions (in 6 patients) and as a nodule in 8 AN in 7 patients. An additional areola was present with nodules in 2 lesions (2 patients). More rare clinical representations of the AN included patch hair in 1 lesion, a macule in 5 instances of the AN (in 5 patients) and a dimpling in 7 lesions (in 6 patients).

\section{Dermoscopy of the accessory nipples}

The dermoscopic findings concerning AN are presented in Table 2 and Figures 5-10. In the case of the absence of central white scar-like areas, a central network-like structure is always present (4/19 lesions). If the central white scar-like area is present (15 lesions), then central network-like structures appear in only $20 \%$ of subjects (3/19 lesions) (Fisher's exact test $p=0.0091)$.

White cobblestone-like structures (7/19 lesions) were also present, with central network-like structures in 5/19 lesions (72\%) (Fisher's exact test $p=0.0449)$. There is no statistical significance between the occurrence of the 
Table 2. The dermoscopic findings concerning accessory nipples in author's own study

\begin{tabular}{llcc}
\hline \multirow{2}{*}{ No. of figure } & \multicolumn{3}{c}{ Dermoscopic findings in analysed accessory nipples } \\
\cline { 2 - 4 } & Total number of analysed dermoscopic pictures of all AN & 19 lesions (100\%) & 14 patients (100\%) \\
\hline Figure 5 & Peripheral network (network-like structures) & 12 lesions (63\%) & 10 patients (71\%) \\
\hline Figure 5 & Central white scar-like area & 15 lesions (79\%) & 12 patients (86\%) \\
\hline Figure 6 & Central network-like structures & 7 lesions (37\%) & 6 patients (43\%) \\
\hline Figure 7 & Cleft-like appearance & 8 lesions (42\%) & 7 patients (50\%) \\
\hline Figure 8 & White cobblestone-like structures & 7 lesions (37\%) & 6 patients (43\%) \\
\hline Figure 9 & Central round dimpling with a plug & 6 lesions (32\%) & 4 patients (29\%) \\
\hline Figure 10 & Fisheye-like structures resembling comedo-like openings & 9 lesions (47\%) & 7 patients (50\%) \\
\hline
\end{tabular}

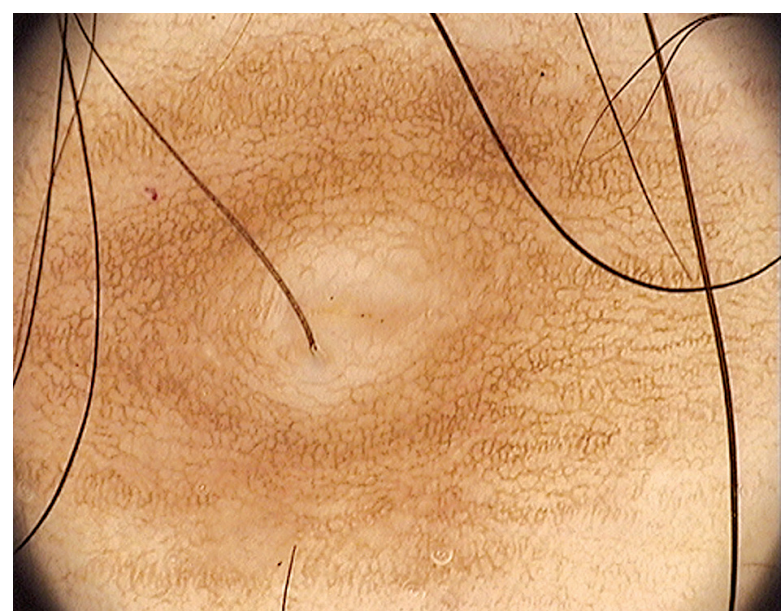

Figure 5. Central white scar-like area. Dermoscopy reveals a centrally located, sharply circumscribed, oval, white area surrounded by a peripheral network (network-like structures). Small dots comparable to these of the fisheye-like structures are also visible in the central part of the lesion, resembling comedo-like openings

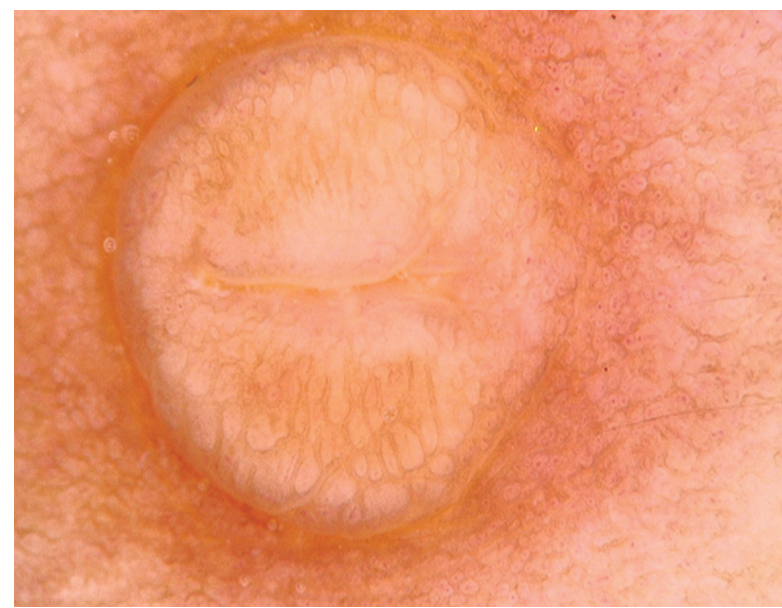

Figure 7. Cleft-like appearance. A cleft dividing the nipple into two nearly equal parts has been observed in the central part of the accessory nipple. The cleft-like appearance corresponds with the tip of the nipple. Moreover, the presence of central network-like structures and the white cobblestone-like pattern have been noted

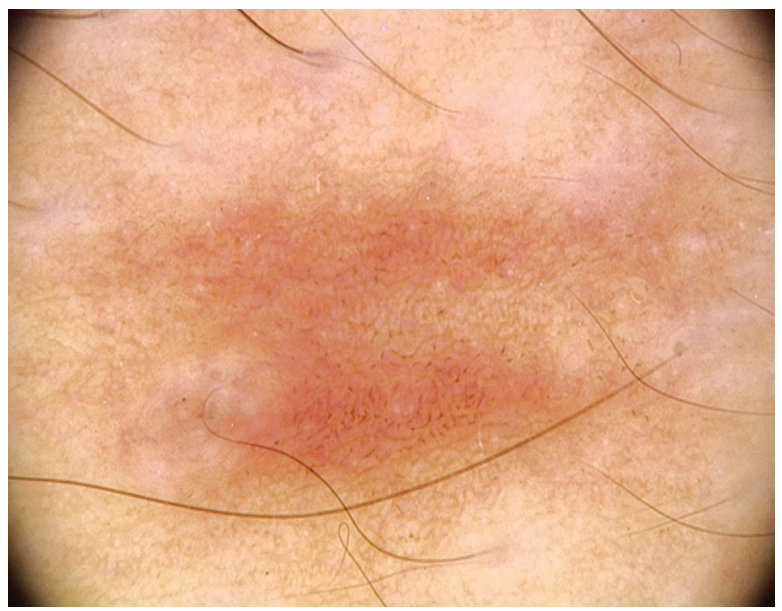

Figure 6. The dermoscopic image shows the presence of central network-like structures covering the whole part of the AN. Numerous, small dark-brown dots, referred to as fisheye-like structures are visible in the central area. These are sharply circumscribed, round structures mimicking comedo-like openings

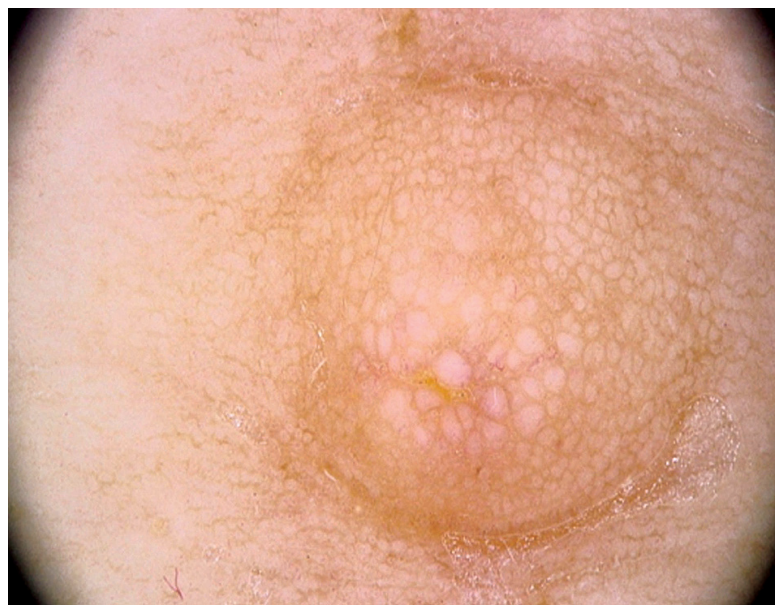

Figure 8. Dermoscopic image of the AN described as a white cobblestone-like pattern. The presence of central network-like structures has been observed within the AN, covering the entire nipple, which divides its background into numerous, closely aggregated, larger, angulated whitish globules resembling cobblestones, described as the white cobblestone-like pattern 


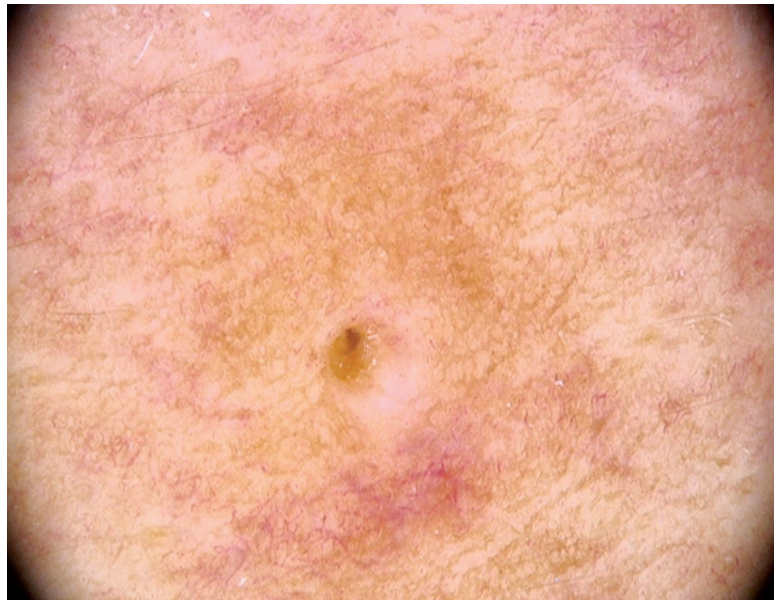

Figure 9. Dermoscopy shows the presence of a central round dimpling with a darker plug and an accompanying central scar-white area surrounded by a peripheral network (network-like structures)

cleft-like appearance and central network-like structures $(p=0.3765)$.

Statistical significance exists between the presence of the central round dimpling and central network-like structures. The occurrence of the central round dimpling never appears alongside central network-like structures in any of the analysed lesions (Fisher's exact test $p=0.0436)$. There is no statistical significance between the presence of central network-like structures and fisheye-like structures $(p=0.7366)$.

The presence of peripheral network-like structures with the occurrence of central scar-like patterns is statistically highly significant (Fisher's exact test $p=0.0091$ ). Central scar-like areas (15/19 lesions) always occurred in the company of peripheral network-like structures (12/19, 80\%).

The presence of peripheral network-like structures $(12 / 19)$ rather excludes the occurrence of white cobblestone-like structures (10 out of 19 lesions were devoid of this dermoscopic feature) ( $p=0.0499)$. There is no statistical significance between the presence of peripheral network-like structures and the cleft-like appearance ( $p=0.3765)$.

The coincidental occurrence of peripheral network-like structures with the central round dimpling is statistically significant. In all the cases of the central round dimpling (6/19 lesions), peripheral network-like structures had always been present $(p=0.0436)$. There is no significant statistical link between peripheral network-like structures and fisheye-like structures ( $p=0.7633$ ).

There is no significant statistical link between central scar-like area and the white cobblestone pattern $(p=0.6026)$. This also applies to the central scar-like area and cleft-like appearance ( $p=0.6026)$ and to the central scar-like area and central round dimpling ( $p=0.2554)$. However, in all cases of the central round dimpling, the

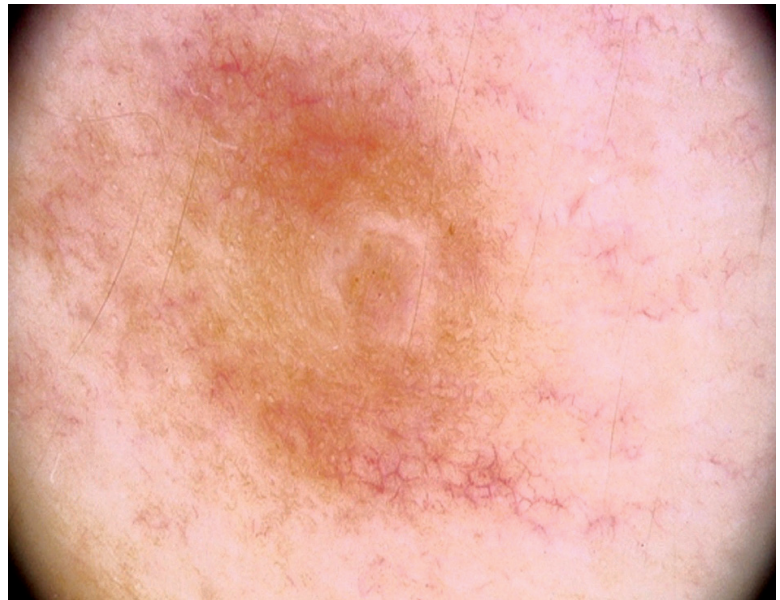

Figure 10. Dermoscopy revealed the presence of a central round dimpling with a darker centre surrounded by a brownish, peripheral network (network-like structures). Fisheye-like structures are visible in the central part of the lesion

occurrence of the central scar-like area had been observed (6/19 lesions). There is no statistical significance between the central scar-like area and fisheye-like structures $(p=0.9056)$.

White cobblestone-like structures with a cleft-like appearance bore no statistical significance $(p=0.0674)$, however, it should be highlighted that in 19 lesions of AN, both white cobblestone and cleft-like structures were absent (11/19 and 9/19, respectively).

The occurrence of the white cobblestone pattern with a central round dimpling was without any statistical significance $(p=0.3331)$. In 6 out of 19 lesions, the presence of a round central dimpling with a plug (84\%) was linked with as many as 5 lesions manifesting the absence of the white cobblestone. There is no statistical significance between fisheye-like and white cobblestone-like structures $(p=0.7633)$.

The presence of the central round dimpling excludes the occurrence of the cleft-like appearance in all lesions $(6 / 19)(p=0.0118)$. The occurrence of fisheye-like structures $(9 / 19)$ in as many as 7 lesions manifested the absence of the cleft-like pattern $(78 \%)(p=0.1698)$. There is no statistical significance between fisheye-like structures and the central round dimpling ( $p=0.3498)$.

The frequency of the occurrence of selected analysed dermoscopic features in the absence of central network-like structures is illustrated in Figure 11 and their frequency in the absence of peripheral network-like structures is described in Figure 12.

\section{Discussion}

The AN is one of extremely rare dermatological conditions. Its frequency amongst the population ranges between $1 \%$ and $2 \%$ [2]. The dermoscopic characteris- 


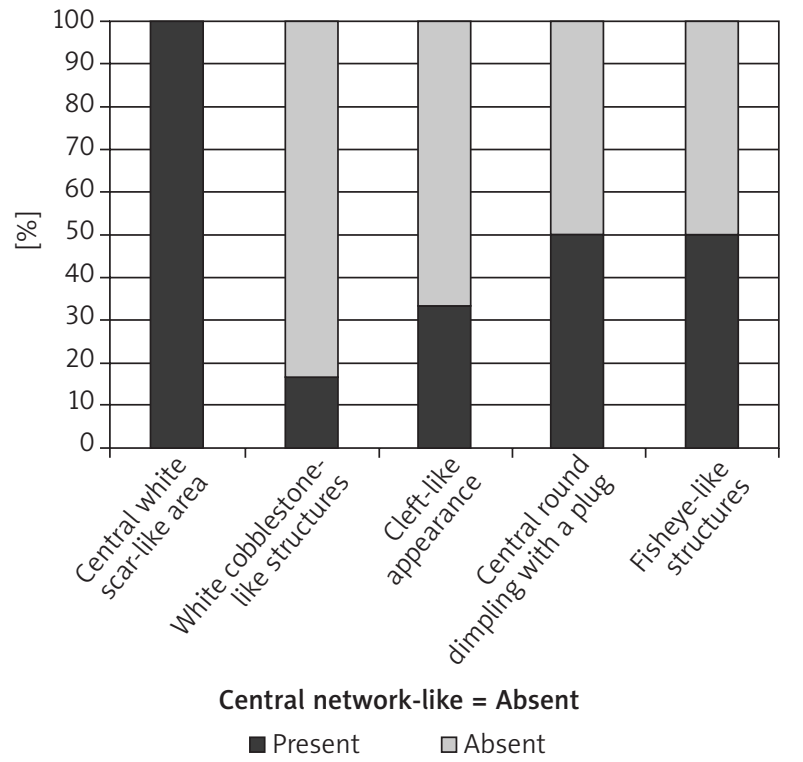

Figure 11. Frequency of occurrence of selected analysed dermoscopic features in the absence of central network-like structures

tics of the AN which have already been defined are: the presence of structures resembling a pigment network (network-like structures) as well as a central white-scar area [5, 11-14]. In our own study, the peripheral network (network-like structures) proved to be present in a total of $79 \%$ of the described changes. The pigment network usually pointed out to a melanocytic character of the changes, according to the algorithm for the differentiation for melanocytic lesions [5]. The pigment network which may at times appear in cases of the AN is in itself an exception in accordance with the algorithm for the differentiation of melanocytic changes. This is due to the fact that it concerns a non-melanocytic change in this type of condition, which is a group to which the AN belongs.

Dermatofibroma is the second exception among the changes characterised by the appearance of a pigment network. This is not classed as a melanocytic lesion, either $[5,11,12,15]$. Moreover, the presence of central scarlike area within the dermatofibroma [15] may also be the basis for incorrect recognition in comparison with the AN [14]. In both exceptions mentioned above, the finding of the pigment network may lead to the erroneous diagnosis of a melanocytic change, which could lead to an unnecessary excision of the diagnosed lesion.

During observations carried out by Zaballos et al. [16] concerning 412 dermatofibromas, a pigment network were observed in $71 \%$ of lesions and white scar-like areas were noted in a total of $57 \%$ of lesions [16]. What is more, the presence of white scar-like areas should always suggest the necessity of ruling out melanoma

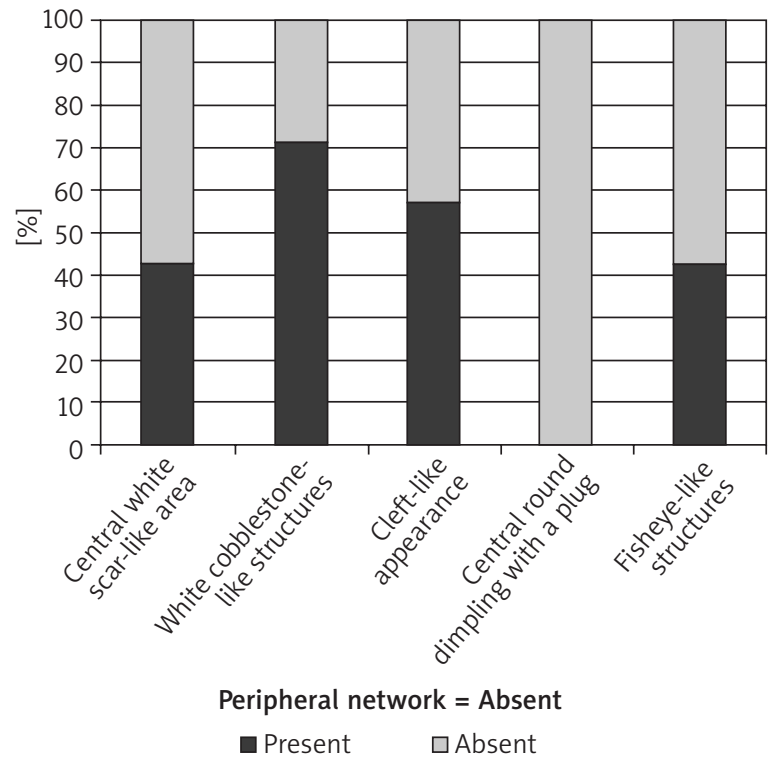

Figure 12. Frequency of occurrence of selected analysed dermoscopic features in the absence of peripheral network (network-like) structures

through a dermoscopic examination - especially its desmoplastic type [17] or the fully regressive melanoma [18]. Scar-like white areas are a usual histological suggestion of fibrosis [3].

The dermoscopic pattern of AN has, until recently, been most commonly described as a peripheral network-like structures [19], as was also the case with central scar-like areas; characteristics of these all belong to the standard dermoscopic features of the AN. Also, the statistical significance of the appearance of the central scar-like area (63\%), as well as of peripheral network-like structures found in $79 \%$ of the analysed lesions has been proven for both of the above characteristics in the course of our own study. To sum up, network-like structures in their central location (7/12) or those placed peripherally $(12 / 12)$ were found in $100 \%$ of the presented cases of the AN.

The new terminology used to describe dermoscopic features found within the AN as that of a white cobblestone-like structure is presented by the authors for the first time in this study. The image does, in fact, in its appearance resemble the previously described classic elevated cobblestone pattern formed by cobblestoned surfaces $[11,12,20]$. This commonly concerns the melanocytic congenital nevi [20]. The cases which the authors have described in the paper were statistically significantly afflicted with such white cobblestone-like structures, along with central network-like structures, which, in a unique way, separated the white, slightly elevated areas of the skin changes, dividing them into structures resembling cobblestones, which commonly appear in the classic dermoscopic cobblestone pattern. 
In Oztas' study [13] concerning 5 cases of the solitary occurrence of the AN, an additional dermoscopic structure was described, referred to as the cleft-like appearance. According to Oztas et al. [13], especially if the central cleft can be demonstrated using dermoscopy, the diagnosis can easily be made, especially in relation to differentiating the condition against dermatofibroma [13]. According to our observations, the cleft-like appearance concerned $42 \%$ of lesions, whereas its presence in all the examined AN was ruled out by the presence of a central round dimpling (31\%). It may thus be summarised on the basis of the conducted dermoscopic analysis that the AN may manifest itself through its cleft-like appearance or through a central round dimpling. The presence of either one of these features was observed in as many as 73\% of lesions. Moreover, peripheral network-like structures were present in all the cases in which we described the central round dimpling.

Fisheye-like structures resembling comedo-like openings were present in $47 \%$ of AN. These are most probably formed of hyperkeratotic masses located within the indentation of the AN. Typically comedo-like openings are most commonly found in seborrheic keratosis and in the dermal nevi, usually of their exophytic type [11, 12, 21].

The knowledge of dermoscopic features concerning skin cancer and melanoma in its early stages [22, 23], and also of rare dermatological disorders, such as the AN and the nevus comedonicus [24] is extremely useful in daily medical practice.

\section{Conclusions}

The conducted dermoscopic analysis of a total of 19 AN confirmed the presence of a pigment network in all the examined lesions (central or peripheral network-like structures), with the peripheral type being the most commonly observed. Also, peripheral network-like structures with the occurrence of a central scar-like area have been most commonly presented in our study. The appearance of the central network-like structures usually meant the presence of white cobblestone-structures in the dermoscopic images of AN. The new dermoscopic structures present in AN were as follows: a central round dimpling with a plug and fisheye-like structures resembling comedo-like openings.

\section{Conflict of interest}

Authors declare no potential conflict of interest. Funding sources: none.

\section{References}

1. Miedziński F. Common integument. In: Human anatomy. Peripheral nervous system. Autonomic nervous system. Common integument. Sense organs [Polish]. Bochenek A, Reicher M (eds). PZWL, Warsaw 1989; 338-43.
2. Wienecke R. Malformations and genetic disorders. In: Braun Falco Dermatology [Polish]. Burgdorf WHC, Plewig G, Wolff HH, Landthaler M (eds). Czelej, Lublin 2010; 775.

3. Goyal T, Bakshi SK, Varshney A. Seven nipples in a male: world's second case report. Indian J Hum Genet 2012; 18: 373-5.

4. Kajava Y. The proportions of supernumerary nipples in the Finnish population. Duodecim 1915; 1: 143-70.

5. Scope A, Benvenuto-Andrade C, Agero AL, et al. Nonmelanocytic lesions defying the two-step dermoscopy algorithm. Dermatol Surg 2006; 32: 1398-406.

6. Grimshaw EC, Cohen PR. Supernumerary nipple and seminoma: case report and review of polythelia and genitourinary cancers. Dermatol Online J 2013; 19: 4.

7. Goedert JJ, McKeen EA, Fraumeni JF Jr. Polymastia and renal adenocarcinoma. Ann Intern Med 1981; 95: 182-4.

8. Rajan S, Munot K, Haselden J. Breast cancer associated with an accessory nipple. BMJ Case Rep 2011; pii: bcr0620114391.

9. Carmichael AR, McIntyre M, Chetty U. Malignant melanoma in accessory nipple in a male. J Coll Physicians Surg Pak 2006; 16: 799-800.

10. Martin VG, Pellettiere EV, Gress D, et al. Paget's disease in an adolescent arising in a supernumerary nipple. J Cutan Pathol 1994; 21: 283-6.

11. Argenziano G, Soyer HP, Chimenti S, et al. Dermoscopy of pigmented skin lesions: results of a consensus meeting via the Internet. J Am Acad Dermatol 2003; 48: 679-93.

12. Soyer HP, Argenziano G, Chimenti S, et al. Dermoscopy of pigmented skin lesions. Eur J Dermatol 2001; 11: 270-6.

13. Oztas MO, Gurer MA. Dermoscopic features of accessory nipples. Int J Dermatol 2007; 46: 1067-8.

14. Blum A, Roehm S. Accessory nipple looks like dermatofibroma in dermoscopy. Arch Dermatol 2003; 139: 948-9.

15. Kilinc Karaarslan I, Gencoglan G, Akalin T, et al. Different dermoscopic faces of dermatofibromas. J Am Acad Dermatol 2007; 57: 401-6.

16. Zaballos P, Puig S, Llambrich A, et al. Dermoscopy of dermatofibromas: a prospective morphological study of 412 cases. Arch Dermatol 2008; 144: 75-83.

17. Debarbieux S, Ronger-Salve S, Dalle S, et al. Dermoscopy of desmoplastic melanoma: report of six cases. Br J Dermatol 2008; 159: 360-3.

18. Lallas A, Apalla Z, Moscarella E, et al. Extensive regression in pigmented skin lesions: a dangerous confounding feature. Dermatol Pract Concept 2012; 2: 202a08.

19. Cabo H, Stolz W. Pigment network: a clue to dermatoscopic diagnosis of melanocytic lesions - supernumerary nipple: another exception to the rule. Dermatol Surg 2004; 30: 1068-9.

20. Zalaudek I, Manzo M, Savarese I, et al. The morphologic universe of melanocytic nevi. Semin Cutan Med Surg 2009; 28: 149-56.

21. Elgart GW. Seborrheic keratoses, solar lentigines, and lichenoid keratoses. Dermatoscopic features and correlation to histology and clinical signs. Dermatol Clin 2001; 19: 347-57.

22. Mackiewicz-Wysocka M, Bowszyc-Dmochowska M, Strzelecka-Węklar D, et al. Basal cell carcinoma - diagnosis. Contemp Oncol (Pozn) 2013; 17: 337-342.

23. Kamińska-Winciorek G, Właszczuk P, Wydmański J. "Mistletoe sign": probably a new dermoscopic descriptor for melanoma in situ and melanocytic junctional nevus in the inflammatory stage. Postep Derm Alergol 2013; 30: 316-9.

24. Kamińska-Winciorek G, Spiewak R. Dermoscopy on nevus comedonicus: a case report and review of the literature. Postep Derm Alergol 2013; 30: 252-4. 